\title{
Tunable SESAM-based mode-locked soliton fiber laser in linear cavity by axial-strain applied to a FBG
}

\author{
I. Aporta, D. Leandro, M. A. Quintela, R.A. Pérez-Herrera, M. Lopez-Amo, J. M. López- \\ Higuera
}

\begin{abstract}
A self-started stable tunable mode-locked (ML) soliton fiber laser in a linear cavity structure composed by commercial passive elements is presented in this paper. It is based on the combination of an Erbium doped fiber (EDF) as the active medium, and a saturated fiber Bragg grating (FBG) as a partial mirror of the cavity. The other side of the cavity consists of a semiconductor saturable absorber mirror (SESAM) acting also as the mode-locking device. The central wavelength of the FBG is selected by the application of axial strain giving rise to a tunable soliton over $8.6 \mathrm{~nm}$ on the $\mathrm{C}$ band with a variable spacing of $0.01 \mathrm{~nm}$. The laser delivers $18.9 \mathrm{ps}$ long pulses with a $0.14 \mathrm{~nm}$ bandwidth, an $8.2 \mathrm{MHz}$ repetition rate, a pulse energy of $89 \mathrm{pJ}$ and a peak power of $4.71 \mathrm{~W}$.
\end{abstract}

Index Terms-Optical fiber lasers, Mode locked lasers, Laser tuning, Optical solitons, Fiber nonlinear optics, Fiber Bragg gratings.

\section{INTRODUCTION}

$\mathrm{I}$ $\mathrm{N}$ the last decade, passively mode-locked fiber lasers have been widely investigated because of the research interest and its applications [1-4]. This type of fiber lasers have been experimentally demonstrated as an alternative to solid-state lasers because of their low cost, compact cavity structure, heat dissipation properties, free-alignment and overall high stability [5-9]. Furthermore, active mode-locked fiber lasers also require additional switching electronics and complex driven modulators [10-12]. Particularly, the capability of passively mode-locked fiber lasers to generate ultrafast solitons are of important interest in practical applications such as optical fiber sensing, biomedical diagnostics, material processing, nonlinear optics, terahertz generation and ultrahigh speed communications [13-15]. In the field of fiber lasers, numerous types of techniques have been proposed to achieve soliton

Manuscript received. This work was supported by the Spanish Comisión Interministerial de Ciencia y Tecnología within project TEC2016-76021-C2 $\mathrm{R}$ and Feder funds and the Spanish Ministry of Education and Culture, within the scholarship FPU14/02196.

I. Aporta is with the Photonics Engineering Group, Universidad de Cantabria, 39005 Santander, Spain (e-mail: aportai@unican.es)

M. A. Quintela and J. M. Lopez-Higuera are with the Photonics Engineering Group, CIBER-bbn and IDIVAL, Universidad de Cantabria, 39005 Santander, Spain (e-mail: angeles.quintela@unican.es; miguel.lopezhiguera@unican.es)

D. Leandro, R.A. Pérez-Herrera and M. López-Amo are with the Electrical and Electronic Engineering department and ISC, Public University of Navarra, 31006 Pamplona, Spain (e-mail: daniel.leandro@unavarra.es; rosa.perez@unavarra.es; mla@unavarra.es) generation and operation, namely nonlinear polarization rotation, nonlinear optical loop mirror, carbon nanotubes, grapheme layer and semiconductor saturable absorber mirrors (SESAM) [16-21]. SESAM-based mode-locking is a wellknown technique with technological maturity and wide range of applicability in commercial lasers. Moreover, the SESAM is more frequently used as a commercial option due to its flexibility and high stability, allowing self-starting modelocking and ultra-short pulse duration in Erbium-doped and Ytterbium-doped fiber lasers [22, 23].

The different pulse formation mechanisms depend on the cavity net dispersion regime and have been widely investigated, including conventional soliton (CS), dissipative soliton, stretched pulse and self-similar pulse [24-27]. Modelocked erbium fiber lasers without additional dispersion compensation often operate in the anomalous dispersion regime, where the combination of nonlinear effects and negative group-velocity dispersion (GVD) generates optical solitons [1]. Usually, the conventional soliton has a constant phase shift over time, leading to a non-chirp or spectral broadening and exhibiting a sech ${ }^{2}$ temporal profile [28], and it operates on picoseconds and sub-picoseconds regime [29,30]. Besides, the conventional soliton lasers operating on stable mode locking regime have been deeply researched, giving rise to different kinds of mode-locking operation, such as boundstate solitons, noise-like pulse and high-order harmonic [31, 32]. Nevertheless, most of research is focused on soliton fiber lasers with short length cavities of tens of meters where the cavity net dispersion varies between -1 and $1 p^{2}$.

On the other hand, the mentioned saturable absorber devices have broadband saturable absorption and can be used to achieve tunable ML fiber lasers over the communication band of interest [33, 34]. Typically, most of the research about tunable erbium-doped ML fiber lasers has been achieved in ring cavities, which need additional components as an attenuator, interferometer, tunable filter or similar to perform a wavelength-tunable ML structure [35, 36]. In comparison with ring cavities, linear ones allow much simpler design which translates into lower cost. In this sense, fiber Bragg gratings can be employed in an all-fiber linear cavity structure with all its advantages as free-alignment, low insertion loss and ease of handling [37]. For this reason, an FBG has been selected as a mirror and as the element of wavelength selection through the application of controlled strain in a linear cavity. 
In the present work, a new linear cavity-based self-started tunable mode-locked soliton fiber laser structure is experimentally demonstrated. The cavity is constructed by employing an FBG as a partial mirror, EDF as the active medium, a polarization controller (PC) in combination with PM fiber to control the polarization states, and a SESAM as the saturable absorber mirror. This structure gives rise to a laser cavity with an anomalous group velocity dispersion of -0.04464 $p s^{2}$. Experimental results show a tunable soliton spectrum of $0.14 \mathrm{~nm}$ of bandwidth due to the narrow filtering of the FBG, with a pulse width of 18.9 ps as well as a repetition rate, energy pulse and peak power of $8.2 \mathrm{MHz}, 89$ $\mathrm{pJ}$ and $4.71 \mathrm{~W}$, respectively. The soliton fiber laser operates in the $\mathrm{C}$ band with a variable spacing of $0.01 \mathrm{~nm}$. This structure is simpler than previous ring structures $[38,39]$ and provides a wider tunable band, achieving wavelength control of the soliton over an $8.6 \mathrm{~nm}$ tunable band through the wavelength tuning of the FBG. The proposed fiber laser is totally based on commercial passive elements in a simple all-fiber structure, which ensures an easy construction at low cost for a tunable CS fiber laser. This laser structure is well suited for practical applications, such as optical communications or sensors multiplexing [40].

\section{EXPERIMENTAL SETUP AND PRINCIPLE}

The schematic diagram of the proposed ML soliton fiber laser is depicted in Fig. 1. The linear cavity consists of a saturated FBG, mounted on a manual linear stage, with a 0.32 $\mathrm{nm}$ bandwidth and a reflectivity over $90 \%$, a 6.2 meter-long EDF (Liekki Er-30) with an absorption of $30 \mathrm{~dB} / \mathrm{m}$ at $1530 \mathrm{~nm}$ and a dispersion coefficient of $-9.8 \mathrm{ps} \cdot \mathrm{nm}^{-1} / \mathrm{km}$ at $1550 \mathrm{~nm}$ and a SESAM (Batop $\mathrm{GmbH}$ ) which is butt-coupled and placed in a passive heat sink at the end of the cavity. The SESAM has a modulation depth of $15 \%$, a non-saturable loss of $10 \%$, a saturation fluence of $70 \mathrm{\mu J} / \mathrm{cm}^{2}$ and a relaxation time of $2 \mathrm{ps}$. The other fibers are 4 meters of single mode fiber (SMF-28) and 2.2 meters of polarization maintaining fiber (PM-1550HP) with a dispersion parameter of $17 \mathrm{ps} \cdot \mathrm{nm}^{-1} / \mathrm{km}$ and $17.5 \mathrm{ps} \cdot \mathrm{nm}^{-1} / \mathrm{km}$ at $1550 \mathrm{~nm}$ respectively. The cavity includes a PC to adjust the linear birefringence. The EDF is pumped by a $980 \mathrm{~nm}$ laser diode (LD) with an optical power of $500 \mathrm{~mW}$ via a $980 / 1550 \mathrm{~nm}$ wavelength division multiplexer (WDM). A $980 \mathrm{~nm}$ isolator is coupled between the LD and the WDM to suppress the possible back reflections protecting the pump source. The common port of the WDM is connected to an electric spectrum analyzer (ESA) through a photo-receiver with a bandwidth of $1 \mathrm{GHz}$ (New-focus 1611). The $90 \%$ port of a $90 / 10$ fiber-fuse optical coupler (OC) is engaged to feedback into the linear cavity while the $10 \%$ port provides the laser output. The output signal is divided by a $50 / 50$ coupler to an optical spectrum analyzer (OSA) and an oscilloscope to monitor the output laser simultaneously. The OSA (ADVQ8384) with a $0.02 \mathrm{~nm}$ resolution and the oscilloscope (Infiniium DCA-J 86100C) with a 65 Ghz optical bandwidth are used for spectral and temporal analysis of the ML fiber laser respectively. The ESA (R\&S FPS) in combination with the photo-receiver is used to obtain the RF spectrum of the soliton fiber laser. An EXFO FPM-300 power meter has been used to measure the laser output.

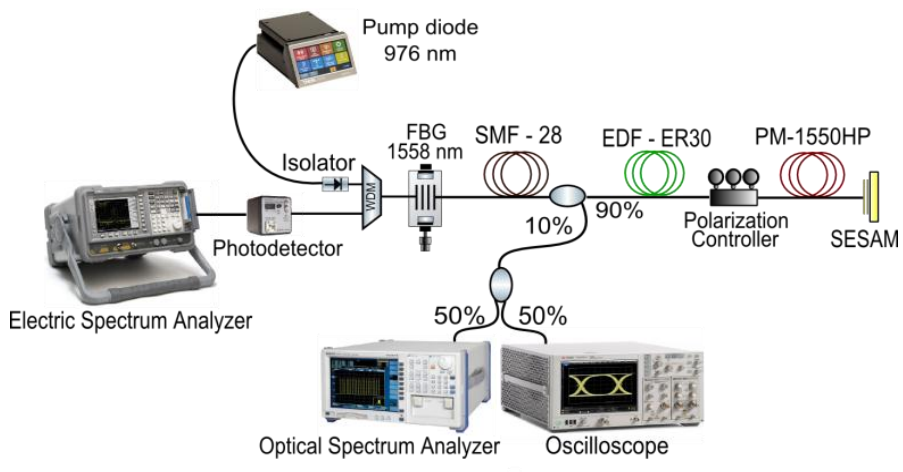

Fig.1 Schematic diagram of the experimental setup for the tunable mode-locked soliton fiber laser

The structure shown in Fig.1 allows soliton generation through forward pumping of the EDF in a cavity of 12.4 meters-long with an anomalous net dispersion regime of $0.04464 \mathrm{ps}^{2}$. Similar ring cavity structures of solitons with these values of anomalous net dispersion have been previously achieved [41, 42].The total net dispersion of the cavity is estimated using the method in [28, 43] and is determined by the N-order position of the Kelly sidebands from the center wavelength of the ML soliton. This theoretical value has been calculated by (1):

$$
\left(\Delta \lambda_{N}\right)^{2}=\frac{2 \lambda_{0}^{2}}{c D L} \cdot N-0.0787 \cdot \frac{\lambda_{0}^{4}}{(c \tau)^{2}}
$$

where $\Delta \lambda_{N}$ is the wavelength distance from the Kelly sideband position to the central wavelength, $\mathrm{N}$ is the sideband order, $\lambda_{0}$ is the soliton central wavelength, $\mathrm{c}$ is the speed of light in vacuum, $\mathrm{D}$ is the dispersion parameter, $\mathrm{L}$ is the cavity length and $\tau$ is the soliton pulse width. The wavelength emission is determined by the saturated FBG shown in Fig.2. This saturated FBG has a broadened spectrum and a flattened peak which easily-drive the soliton generation process at lower pump powers, unlike conventional FBGs with narrow bandwidth and sharpened peak. The pulse duration of the soliton is limited by the reflection bandwidth of the FBG and it has been employed not only as a wavelength-selector, but also implies a spectral filtering effect. The self-started ML soliton is easily obtained by controlling the rotation of the PC and matching the polarization state that leads to a stabilized pulsed laser.

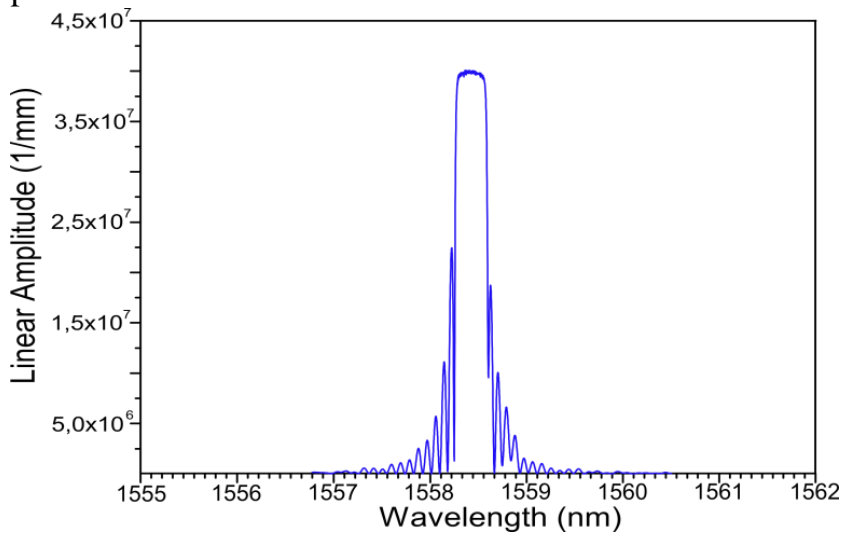

Fig.2 Optical spectrum of the saturated FBG 
The ML soliton tunability is achieved by the wavelength selection variability of the FBG through the application on controlled axial-strain as in [44]. The manual linear stage is used to position and strain the FBG. This manner, the wavelength of the pulsed fiber laser is setting, performing a tunable soliton whose temporal characteristics are hardly affected by the spectral shift. Only the extremely low changes in the dispersion parameter through the variability of the wavelength emission generate little differences in the temporal pulse width of the laser (which are negligible). The selfstarting condition, the temporal response and the spectral distribution of ML soliton are maintained through the tunable band and have been experimentally contrasted.

\section{RESULTS AND DISCUSSION.}

In this experimental setup, continuous wave (CW) operation has been realized at $10.2 \mathrm{~mW}$ of pump power. The single pulse self-started ML soliton operation can be observed when the pump power is $29.6 \mathrm{~mW}$ by appropriately adjusting the polarization state of the linear cavity through the PC. The soliton output spectrum is shown in Fig.3a) when the pump power is $\sim 40 \mathrm{~mW}$ and is centered at $1558.4 \mathrm{~nm}$. The CS bandwidth is only $\sim 0.14 \mathrm{~nm}$ and is limited by the reflection spectrum of the FBG. As it can be seen, the soliton spectrum exhibits the typical Kelly sidebands only in one side, because the filtering of the FBG cuts off the spectrum. Accordingly, the generation of the sidebands is suppressed [45]. The average output power of the pulses is about $0.73 \mathrm{~mW}$ and the ouput pulse energy is calculated to be 89 pJ. Fig.3b) illustrates the corresponding temporal trace of the soliton. The full width at half maximum (FWHM) is $28.9 \mathrm{ps}$ and the corresponding $\operatorname{sech}^{2}$ fit profile yields an estimated pulse duration of $18.9 \mathrm{ps}$. The time bandwidth product (TBP) is close to the transform limit (0.315) of $\operatorname{sech}^{2}$-shaped pulse with a value of 0.327 indicating that the pulse is nearly free-chirp because of the short anomalous net dispersion. The pulse train can be observed in the oscilloscope trace of Fig.3c). The separation between adjacent pulses is $121.95 \mathrm{~ns}$, which corresponds to the round trip time of the cavity and a repetition rate of 8.2 MHz. Fig 3d) illustrates the RF spectrum of the ML soliton laser measured under a bandwidth resolution of $10 \mathrm{KHz}$. The fundamental repetition rate is determined by the 12.4 meterslong cavity and the theoretical result is calculated in (2):

$$
f_{\text {rep }}=\frac{c}{2 L \cdot n}
$$

where $\mathrm{L}$ is the total length of the cavity, $\mathrm{c}$ is the speed of light in vacuum and $n$ is the effective refractive index of the fiber. The signal-to-noise ratio (SNR) of the RF spectrum is $\sim 50 \mathrm{~dB}$, which indicates a good mode-locking stability. No spectrum modulation is observed in the $\sim 350 \mathrm{MHz}$ wideband $\mathrm{RF}$ spectrum shown in Fig.3d), implying no Q-switching instabilities
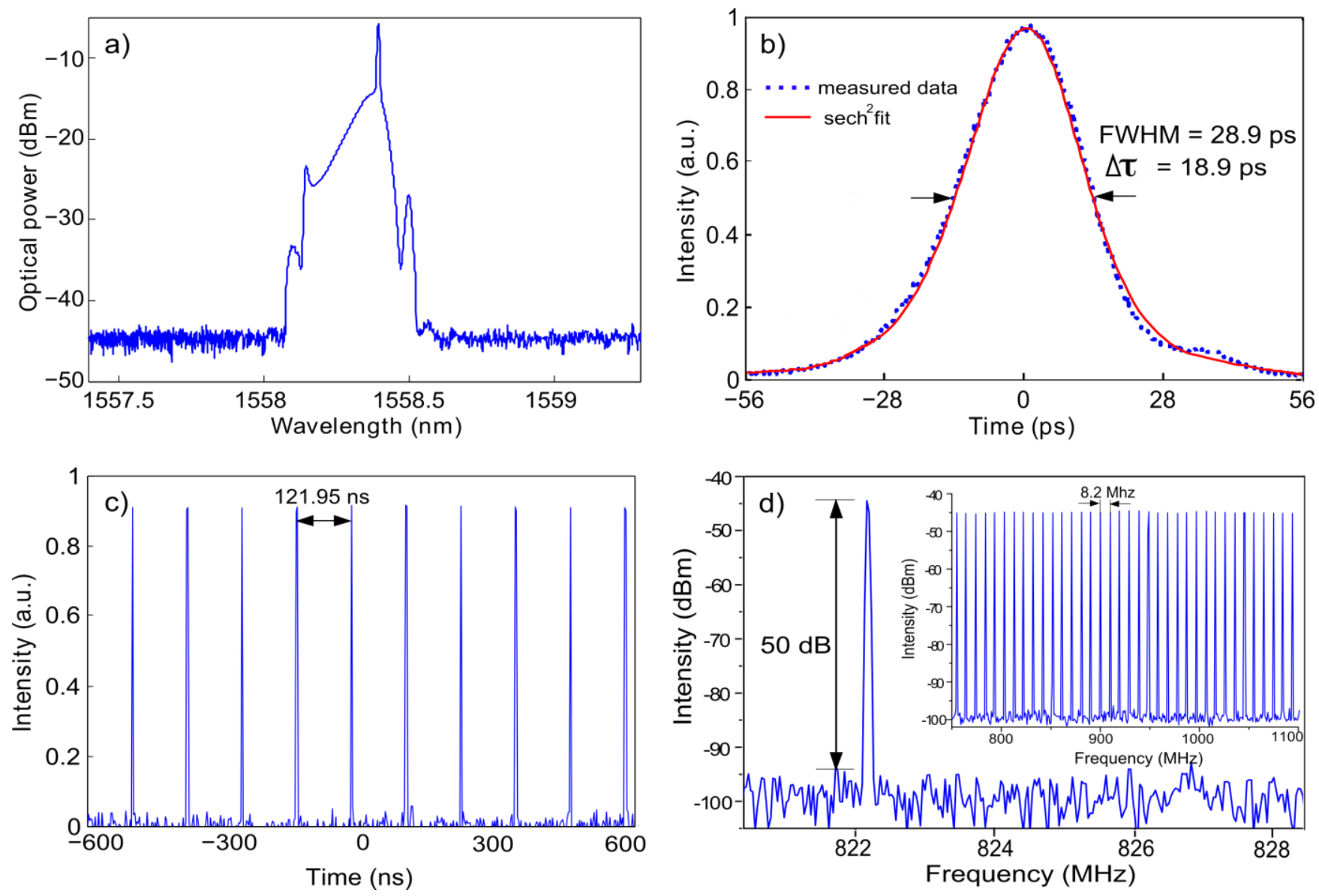

Fig.3 a) Optical spectrum, b) temporal trace, c) oscilloscope trace and d) RF spectrum of CS in the ML fiber laser. 


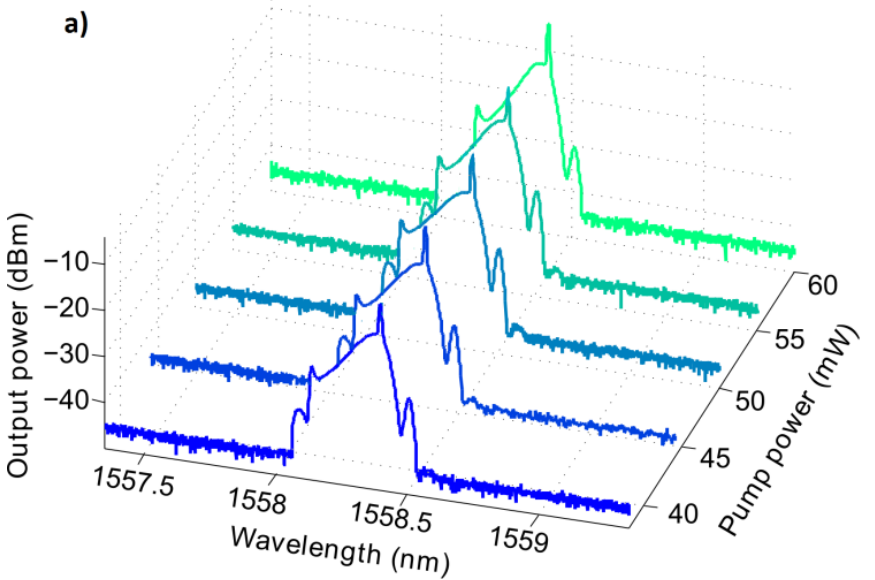

Fig.4 a) Optical output spectra and b) temporal traces of the

Fig. 4a) shows the output spectra of the ML soliton fiber laser for different pump powers. As it can be seen, the shapes of the laser in the optical domain barely change in the optical spectrum. The FWHM of the laser remains almost constant with increased pump power, despite of the improved optical gain of the oscillator and the increase of synchronized resonating modes [20]. Furthermore, the selected erbiumdoped fiber generates a gain bandwidth that reaches its maximum with a pumping power of $\sim 80 \mathrm{~mW}$. The laser output peak power of the different sampled spectra has also been measured showing liner changes. The central wavelength emission fixed and strain-controlled by the FBG keeps unchanged with increasing power.

The temporal traces of the ML soliton corresponding to their optical spectra are depicted in Fig. 4b). As it can be observed, the pulse duration of the different sampled CS remains almost constant and its variation is inversely proportional to its spectral widening. As the limit of the stable ML range approaches, a slight broadening in the frontal tail of the pulse is observed. This phenomenon is mainly due to two reasons: the first is related to the temporal behavior of SESAM. The temporal relaxation of SESAM is approximately 2 ps but presents a different temporal response at high fluence [46].

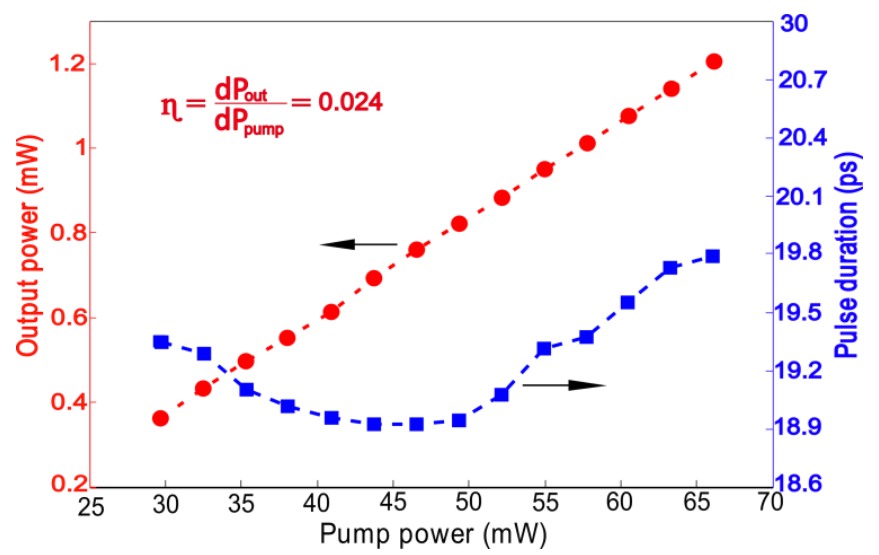

Fig.5 Optical average output power and pulse duration of the ML soliton over the whole stable ML regime.

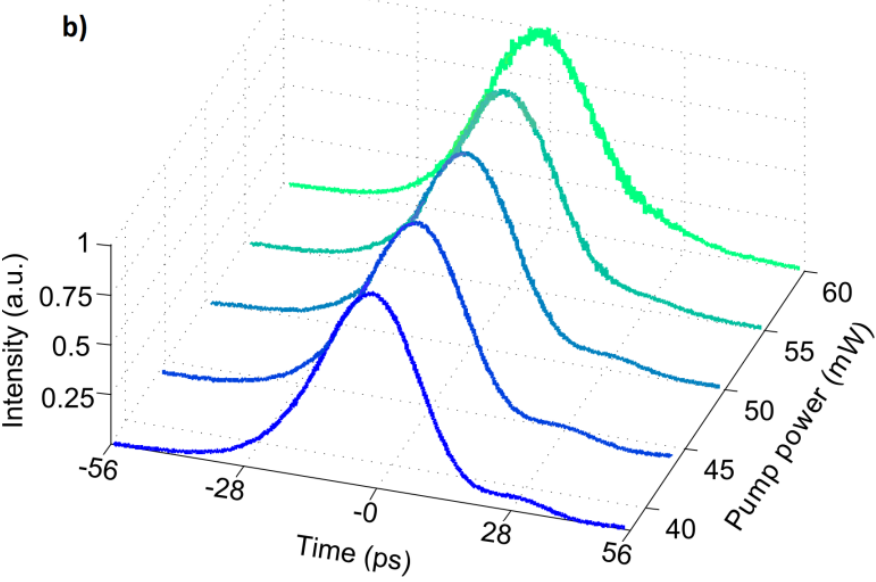

ML soliton centered at $1558.4 \mathrm{~nm}$ for different pump powers

This effect could broaden the pulses, but in this case, the spectral filtering of the FBG induces a greater temporal broadening than the one produced by the bi-temporal behavior of the SESAM, keeping it temporary imperceptible. Another reason is the frequency chirp nonlinearities derived from the increase of the peak power where the balance between nonlinear-effects and dispersion is perturbed [47]. Moreover, when the SESAM is reaching the limit of the ML stable regime, the pulses become noisy just before entering in the multi-pulse operation regime. This could be due to unstable excitation of higher order solitons by amplitude fluctuations or because of the beating between the pulse and optical noise from the erbium-doped fiber amplifier [48]. As shown in Fig.4b) the pulses measured at $54.9 \mathrm{~mW}$ and $60.5 \mathrm{~mW}$ are a little bit noisy and slightly broadened because of the effects mentioned before. To contribute to the research, the average output power and pulse duration of the soliton fiber laser for the different pumping powers in the stable ML regime is illustrated in Fig.5. The linear fitting of the red colored average output power implies a linear relationship with the pump power, and the efficiency of the ML laser has been measured to be $2.4 \%$. This is restricted by the losses of the cavity and could be optimized through the selection of a highdoped erbium fiber. The maximum average output power in stable operation obtained in this experimental setup is 1.196 $\mathrm{mW}$ and it is limited by the parameters of the SESAM and its photo-damage threshold. On the other hand, the pulse duration is depicted in blue color and shows the changing tendency of the temporal response of the laser. As it can be observed, when the pump power is over $29.6 \mathrm{~mW}$ and the laser goes into ML regime, the single pulse duration is $19.4 \mathrm{ps}$ and decays to $18.9 \mathrm{ps}$ in the pump power range from $41 \mathrm{~mW}$ to $50 \mathrm{~mW}$. This temporal behavior is due to the spectral broadening occurred with the increased pump power, so the pulse duration narrowing process of the laser is inversely proportional to its spectral broadening. When the pump power is over $50 \mathrm{~mW}$, the pulse duration broadens from 18.9 ps to $19.8 \mathrm{ps}$ due to aforementioned frequency chirp nonlinearities. 


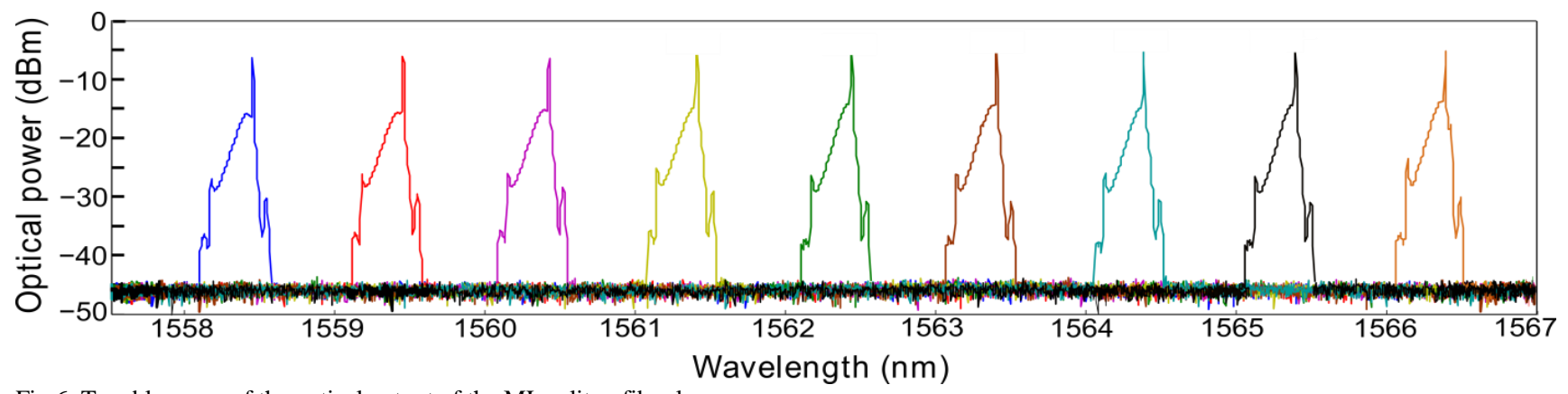

Fig.6. Tunable range of the optical output of the ML soliton fiber laser.

The output spectra of the wavelength tunable ML soliton fiber laser are represented in Fig.6. The OSNR, the pulse duration and the spectral width of the pulsed laser are almost unchanged through the whole tunable range, showing a value of $\sim 40 \mathrm{~dB}, \sim 19 \mathrm{ps}$ and $\sim 0.14 \mathrm{~nm}$ respectively, indicating the stability of the output laser. The mode-locking self-started condition is maintained through the tunable range without modifying the polarization state of the cavity using the PC. The measured wavelength range from $1558.4 \mathrm{~nm}$ to $1567 \mathrm{~nm}$ presents a tunable soliton laser over an $8.6 \mathrm{~nm}$ band with a variable spacing of $0.01 \mathrm{~nm}$. The wavelength is selected through the variation of strain applied to the FBG which is positioned at a mechanical linear stage. This linear stage contains two micro-positioners placed on an optical table that allow strain-control with $0.1 \mu \mathrm{m}$ precision. In this manner, the experimental setup is able to shift the wavelength emission of the laser by varying the axial-strain applied to the FBG, being the wavelength range limited by the rupture of the fiber grating. Previous works on ring structures present different tunable structures but offer some restrictions. One of the main limitations is the dependence of using different passive devices as interferometers or band-pass filters to achieve the tunable lasing, increasing the cost of the structure. The presented experimental set-up can provide $\sim 9 \mathrm{~nm}$ optical band range for the tunable ML soliton emission in a very simple and easy to handle structure. The tuning interval can be swept across the entire C-band selecting the suitable FBG, taking conventional soliton lasers to the stage of practical applications.

\section{CONCLUSIONS}

A self-started stable tunable ML soliton fiber laser in linear cavity has been proposed and demonstrated by combining a conventional EDFA with a SESAM to generate mode-locking and an FBG for wavelength emission tuning. The overall tunable range in this experiment is $8.6 \mathrm{~nm}$ and has been achieved via adjusting mechanical strain applied to an FBG $(1558.4-1567 \mathrm{~nm})$. This range could be increased up to about $\sim 18 \mathrm{~nm}$, by means of using an additional FBG in cascade with the previous one and a polarization controller between the two fiber gratings in order to adjust cavity losses at different wavelengths [49]. The herein described ML soliton fiber laser has low power threshold less than $30 \mathrm{~mW}$, high SNR up to 50 $\mathrm{dB}$, as well as a pulse duration of $18.9 \mathrm{ps}$, a repetition rate of $8.2 \mathrm{MHz}$, a pulse energy of $89 \mathrm{pJ}$ and a peak power of $4.71 \mathrm{~W}$. This all-fiber system has as advantages a lower cost of implementation as well as a wider tunable bandwidth when compared to other ring configurations based on axial-strain applied to an FBG. This compact and simple structure is based on commercial passive devices and is appealing for its practical applications, particularly fiber-optics sensing, spectroscopy, biomedical research or optical communications.

\section{ACKNOWLEDGMENT}

Thanks are due to David Erro, Dr. Santiago Tainta and Jon Mariñelarena for their help and support with this experimental setup.

\section{REFERENCES}

[1] X. Liu, "Soliton formation and evolution in passively-mode-locked lasers with ultralong anomalous-dispersion fibers," Physical Review A, vol. 84, p. 023835, 2011.

[2] M. E. Fermann and I. Hartl, "Ultrafast fiber laser technology," IEEE Journal of Selected Topics in Quantum Electronics, vol. 15, pp. 191-206, 2009.

[3] O. Okhotnikov, A. Grudinin, and M. Pessa, "Ultra-fast fibre laser systems based on SESAM technology: new horizons and applications," New journal of physics, vol. 6, p. 177, 2004.

[4] U. Keller, "Recent developments in compact ultrafast lasers," Nature, vol. 424, pp. 831-838, 2003.

[5] X. Liu, X. Yang, F. Lu, J. Ng, X. Zhou, and C. Lu, "Stable and uniform dual-wavelength erbium-doped fiber laser based on fiber Bragg gratings and photonic crystal fiber," Optics Express, vol. 13, pp. 142-147, 2005.

[6] X. Liu, "Broad and tunable multiwavelength fiber laser at the assistance of modulation-instability-assisted four-wave mixing," Laser Physics, vol. 20, pp. 842-846, 2010.

[7] L. Wang, X. Liu, Y. Gong, D. Mao, and X. Li, "Transitional and steady mode-locking evolution of dissipative solitons," Applied Optics, vol. 49, pp. 2665-2669, 2010.

[8] F. W. Wise, A. Chong, and W. H. Renninger, "High-energy femtosecond fiber lasers based on pulse propagation at normal dispersion," Laser \& Photonics Reviews, vol. 2, pp. 58-73, 2008.

[9] D. Mao, X. Liu, L. Wang, H. Lu, and H. Feng, "Generation and amplification of high-energy nanosecond pulses in a compact allfiber laser," Optics express, vol. 18, pp. 23024-23029, 2010.

[10] H. Chen, S.-P. Chen, Z.-F. Jiang, K. Yin, and J. Hou, "All fiber actively mode-locked ytterbium-doped laser with large range temporal tunability," IEEE Photon. Technol. Lett., vol. 26, pp. 1786-1789, 2014.

[11] H. Chen, S.-P. Chen, Z.-F. Jiang, and J. Hou, "Flexible rectangular wave-breaking-free pulse generation in actively mode-locked ytterbium-doped fiber laser," Optics express, vol. 22, pp. 2644926456, 2014.

[12] C. Kneis, B. Donelan, A. Berrou, I. Manek-Hönninger, T. Robin, B. Cadier, et al., "Actively mode-locked Tm 3+-doped silica fiber laser with wavelength-tunable, high average output power," Optics letters, vol. 40, pp. 1464-1467, 2015.

[13] D. Mao, X. Liu, L. Wang, X. Hu, and H. Lu, "Partially polarized wave-breaking-free dissipative soliton with super-broad spectrum in a mode-locked fiber laser," Laser Physics Letters, vol. 8, p. 134, 2010. 
[14] X. Liu, Y. Cui, D. Han, X. Yao, and Z. Sun, "Distributed ultrafast fibre laser," Scientific reports, vol. 5, p. 9101, 2015.

[15] S. Kobtsev, S. Kukarin, and Y. Fedotov, "Mode-locked Yb-fiber laser with saturable absorber based on carbon nanotubes," Laser Physics, vol. 21, pp. 283-286, 2011.

[16] Y. Zhong, Z. Zhang, and X. Tao, "Passively mode-locked fiber laser based on nonlinear optical loop mirror with semiconductor optical amplifier," Laser physics, vol. 20, pp. 1756-1759, 2010.

[17] S. Smirnov, S. Kobtsev, S. Kukarin, and A. Ivanenko, "Three key regimes of single pulse generation per round trip of all-normaldispersion fiber lasers mode-locked with nonlinear polarization rotation," Optics express, vol. 20, pp. 27447-27453, 2012.

[18] B. Xu, A. Martinez, S. Y. Set, C. S. Goh, and S. Yamashita, "A net normal dispersion all-fiber laser using a hybrid mode-locking mechanism," Laser Physics Letters, vol. 11, p. 025101, 2013.

[19] C. Zeng, X. Liu, and L. Yun, "Bidirectional fiber soliton laser mode-locked by single-wall carbon nanotubes," Optics express, vol. 21, pp. 18937-18942, 2013.

[20] Y.-H. Lin, C.-Y. Yang, J.-H. Liou, C.-P. Yu, and G.-R. Lin, " sing graphene nano-particle embedded in photonic crystal fiber for evanescent wave mode-locking of fiber laser," Optics express, vol. 21, pp. 16763-16776, 2013.

[21] L. Zhang, J. Zhou, Z. Wang, X. Gu, and Y. Feng, "SESAM modelocked, environmentally stable, and compact dissipative soliton fiber laser," IEEE Photonics Technology Letters, vol. 26, pp. 13141316, 2014.

[22] A. Agnesi, L. Carrá, F. Pirzio, R. Piccoli, and G. Reali, "Low repetition rate, hybrid fiber/solid-state, $1064 \mathrm{~nm}$ picosecond master oscillator power amplifier laser system," JOSA B, vol. 30, pp. 2960-2965, 2013.

[23] M. Wang, C. Chen, Q. Li, K. Huang, and H. Chen, "Passively mode-locked Er-doped fiber laser based on a semiconductor saturable absorber mirror," in SPIE/COS Photonics Asia, 2014, pp. 92701E-92701E-6.

[24] Y. Cui, X. Liu, and C. Zeng, "Conventional and dissipative solitons in a CFBG-based fiber laser mode-locked with a graphene-nanotube mixture," Laser Physics Letters, vol. 11, p. 055106, 2014.

[25] K. Krzempek, G. Sobon, P. Kaczmarek, and K. M. Abramski, "A sub-100 fs stretched-pulse $205 \mathrm{MHz}$ repetition rate passively mode-locked Er-doped all-fiber laser," Laser Physics Letters, vol. 10, p. 105103, 2013.

[26] W. H. Renninger, A. Chong, and F. W. Wise, "Self-similar pulse evolution in an all-normal-dispersion laser," Physical Review A, vol. 82, p. 021805, 2010.

[27] Z. Zhang, B. Öktem, and F. Ilday, "All-fiber-integrated solitonsimilariton laser with in-line fiber filter," Optics letters, vol. 37, pp. 3489-3491, 2012.

[28] L. Nelson, D. Jones, K. Tamura, H. Haus, and E. Ippen, "Ultrashort-pulse fiber ring lasers," Applied Physics B: Lasers and Optics, vol. 65, pp. 277-294, 1997.

[29] C. Zhao, Y. Zou, Y. Chen, Z. Wang, S. Lu, H. Zhang, et al., "Wavelength-tunable picosecond soliton fiber laser with topological insulator: $\mathrm{Bi} 2 \mathrm{Se} 3$ as a mode locker," Optics express, vol. 20, pp. 27888-27895, 2012.

[30] D. Popa, Z. Sun, T. Hasan, W. Cho, F. Wang, F. Torrisi, et al., "74-fs nanotube-mode-locked fiber laser," Applied Physics Letters, vol. 101, p. 153107, 2012.

[31] Y.-Q. Huang, Z.-A. Hu, H. Cui, Z.-C. Luo, A.-P. Luo, and W.-C. $\mathrm{Xu}$, "Coexistence of harmonic soliton molecules and rectangular noise-like pulses in a figure-eight fiber laser," Optics Letters, vol. 41, pp. 4056-4059, 2016.

[32] C. Mou, S. Sergeyev, A. Rozhin, and S. Turitsyn, "Polarization dynamics of bound state solitons in a carbon nanotubes mode locked erbium doped fiber laser," in Lasers and Electro-Optics Europe (CLEO EUROPE/IQEC), 2013 Conference on and International Quantum Electronics Conference, 2013, pp. 1-1.

[33] Y. Ozeki and D. Tashiro, "Fast wavelength-tunable picosecond pulses from mode-locked Er fiber laser using an intracavity filter with repetition rate compensator," in Lasers and Electro-Optics (CLEO), 2015 Conference on, 2015, pp. 1-2.

[34] M. Soltanian, I. Amiri, W. Chong, S. Alavi, and H. Ahmad, "Stable dual-wavelength coherent source with tunable wavelength spacing generated by spectral slicing a mode-locked laser using microring resonator," IEEE Photonics Journal, vol. 7, pp. 1-11, 2015.

W. He, M. Pang, and P. S. J. Russell, "Wideband-tunable soliton fiber laser mode-locked at $1.88 \mathrm{GHz}$ by optoacoustic interactions in solid-core PCF," Optics Express, vol. 23, pp. 24945-24954, 2015.

[36] M. Li, X. Zou, J. Qiu, W. Li, and W. Jian, "Tunable multiwavelength dissipative soliton generation in an $\mathrm{Yb}$-doped fiber laser based on SESAM and Lyot-Sagnac filter," in OptoElectronics and Communications Conference (OECC), 2015, 2015, pp. 1-3.

[37] J. Canning, "Fibre gratings and devices for sensors and lasers," Laser \& Photonics Reviews, vol. 2, pp. 275-289, 2008.

[38] C. Zou, T. Wang, Z. Yan, Q. Huang, M. AlAraimi, A. Rozhin, et al., "Wavelength-tunable passively mode-locked Erbium-doped fiber laser based on carbon nanotube and a $45^{\circ}$ tilted fiber grating," Optics Communications, 2017.

[39] Y. Yan, J. Wang, A. P. Zhang, Y. Shen, and H. Tam, "Tunable Lband mode-locked Bi-EDF fiber laser based on chirped Fiber Bragg grating," in Bragg Gratings, Photosensitivity, and Poling in Glass Waveguides, 2016, p. BM3B. 5.

[40] M. Fernandez-Vallejo, D. Ardanaz, and M. López-Amo, "Optimization of the available spectrum of a WDM sensors network using a mode-locked laser," Journal of Lightwave Technology, vol. 33, pp. 4627-4631, 2015.

[41] M. Zhang, E. Kelleher, S. Popov, and J. Taylor, "Ultrafast fibre laser sources: examples of recent developments," Optical Fiber Technology, vol. 20, pp. 666-677, 2014.

[42] Z.-x. Zhang, D. Popa, V. Wittwer, S. Milana, T. Hasan, Z. Jiang, et al., "All-fiber nonlinearity-and dispersion-managed dissipative soliton nanotube mode-locked laser," Applied Physics Letters, vol. 107, p. 241107, 2015.

[43] Y. Ge, Q. Guo, J. Shi, X. Chen, Y. Bai, J. Luo, et al., "Revision on fiber dispersion measurement based on Kelly sideband measurement," Microwave and Optical Technology Letters, vol. 58, pp. 242-245, 2016.

[44] I. A. Litago, R. A. Pérez-Herrera, M. Á. Quintela, M. López-Amo, and J. M. López-Higuera, "Tunable Dual-Wavelength Random Distributed Feedback Fiber Laser With Bidirectional Pumping Source," Journal of Lightwave Technology, vol. 34, pp. 41484153, 2016.

[45] D. Han and X. Liu, "Sideband-controllable mode-locking fiber laser based on chirped fiber Bragg gratings," Optics express, vol. 20, pp. 27045-27050, 2012.

[46] A. Cabasse, B. Ortac, G. Martel, A. Hideur, and J. Limpert, "Highly normal dispersion Er-doped fiber laser mode-locked with a SESAM," in Conference on Lasers and Electro-Optics, 2008, p. CTuFF4.

[47] D. Mao, X. Liu, D. Han, and H. Lu, "Compact all-fiber laser delivering conventional and dissipative solitons," Optics letters, vol. 38, pp. 3190-3193, 2013.

[48] B.-E. Olsson, P. Ohlén, and D. Blumenthal, "Noise in sliced selfphase modulation broadened spectrum," in Lasers and ElectroOptics, 2000.(CLEO 2000). Conference on, 2000, pp. 207-208.

[49] Z.-K. Wang, F. Zou, Z.-W. Wang, S.-T. Du, and J. Zhou, "Tunable and Switchable Narrow Bandwidth Semiconductor-Saturable Absorber Mirror Mode-Locked Yb-Doped Fiber Laser Delivering Different Pulse WidthsSupported by the National High Technology Research and Development Program of China under Grant No 2014AA041901, NSAF Foundation of the National Natural Science Foundation of China under Grant No U1330134, the Opening Project of Shanghai Key Laboratory of All Solid-State Laser and Applied Techniques under Grant No 2012ADL02, and the National Natural Science Foundation of China under Grant Nos 61308024 and 11174305," Chinese Physics Letters, vol. 33, p. $034202,2016$. 